
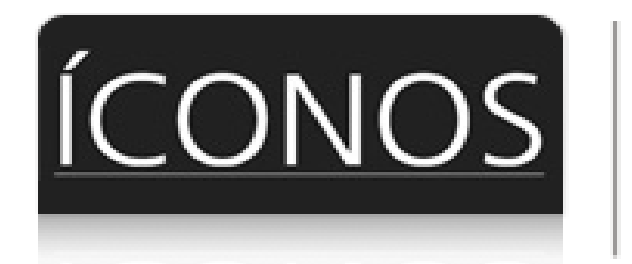

Iconos. Revista de Ciencias Sociales

ISSN: 1390-1249

revistaiconos@flacso.org.ec

Facultad Latinoamericana de Ciencias

Sociales

Ecuador

Falconí, Fander; León G., Mauricio

Pobreza y desigualdad en América Latina

Iconos. Revista de Ciencias Sociales, núm. 15, diciembre, 2002, pp. 96-102

Facultad Latinoamericana de Ciencias Sociales

Quito, Ecuador

Disponible en: http://www.redalyc.org/articulo.oa?id=50901510

- Cómo citar el artículo

- Número completo

- Más información del artículo

- Página de la revista en redalyc.org

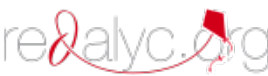

Sistema de Información Científica

Red de Revistas Científicas de América Latina, el Caribe, España y Portugal

Proyecto académico sin fines de lucro, desarrollado bajo la iniciativa de acceso abierto 


\section{Pobreza y desigualdad en América Latina}

\section{diálogo con Rob Vos*}

Fander Falconi***

y Mauricio León G.*****

Rob Vos es economista, profesor de Finanzas y Desarrollo y vicerrector del Instituto de Estudios Sociales de La Haya, Países Bajos. Ha realizado una serie de publicaciones sobre macroeconomía y desarrollo social en Ecuador desde inicios de la década del 80 , y se ha vinculado como asesor a varias instituciones gubernamentales del país como el CONADE, INEC y SIISE. Ha colaborado además, como profesor visitante de FLACSO en diversas oportunidades. Actualmente, coopera con la especialidad de Economía del Desarrollo del Programa de Economía de esta institución académica.

Ha realizado recientemente varias publicaciones sobre pobreza y desigualdad en América Latina, entre las que se destaca: Economic Liberalization, Distribution and Poverty: Latin America in the 1990s, trabajo que efectúa conjuntamente con Enrique Ganuza, Lance Taylor y Ricardo Paes de Barros en el año 2001 (Cheltenham UK, Northampton, MA: Edward Elgar Publishers). En los últimos años, particularmente en el año 2000, han salido a la luz varias de sus investigaciones sobre el Ecuador entre las que constan: Ecuador

* Professor del Institute of Social Studies-The Hague

** Coordinador del Programa de Economía de FLACSO, Ecuador

*** Coordinador (e) del capítulo Ecuador de la Red sobre Desigualdad y Pobreza (NIP, por sus siglas en inglés)
1999: Crisis y Protección Social, estudio que realiza con otros autores. Con Mauricio León desarrolla la investigación La pobreza urbana en el Ecuador: Mitos y realidades. El material bibliográfico ecuatoriano que aquí se menciona contó con el auspicio del SIISE y el sello editorial de Abya-Yala.

Su larga trayectoria como investigador de temas del desarrollo en América Latina y su vasto conocimiento sobre la realidad ecuatoriana hacen del Dr. Rob Vos un referente necesario para entender de mejor manera el desempeño económico y social de la región y el país, en las últimas décadas.

F.F. - M.L.G: ¿Cuál ha sido el impacto general de la liberalización comercial registrada a partir de los años 90 en América Latina, en cuanto a la estructura económica, el mercado de trabajo, la pobreza y la desigualdad?

El resultado más marcado es el aumento de la desigualdad en la distribución de ingresos. Ello sucedió en casi todos los países de la región, de acuerdo a un estudio que se publicó recientemente. ${ }^{1}$ La liberalización comercial ha provocado un cambio tecnológico en sectores importantes de las economías de la región; ha demandado, en particular, más ma-

1 El estudio comprende un análisis de 17 países de la región, fue realizado para PNUD y CEPAL. Ver: Enrique Ganuza, Lance Taylor, Ricardo Paes de Barros y Rob Vos (coord.), 2001, Liberalización, Desigualdady Pobreza: América Latina y el Caribe en los 90, Buenos 


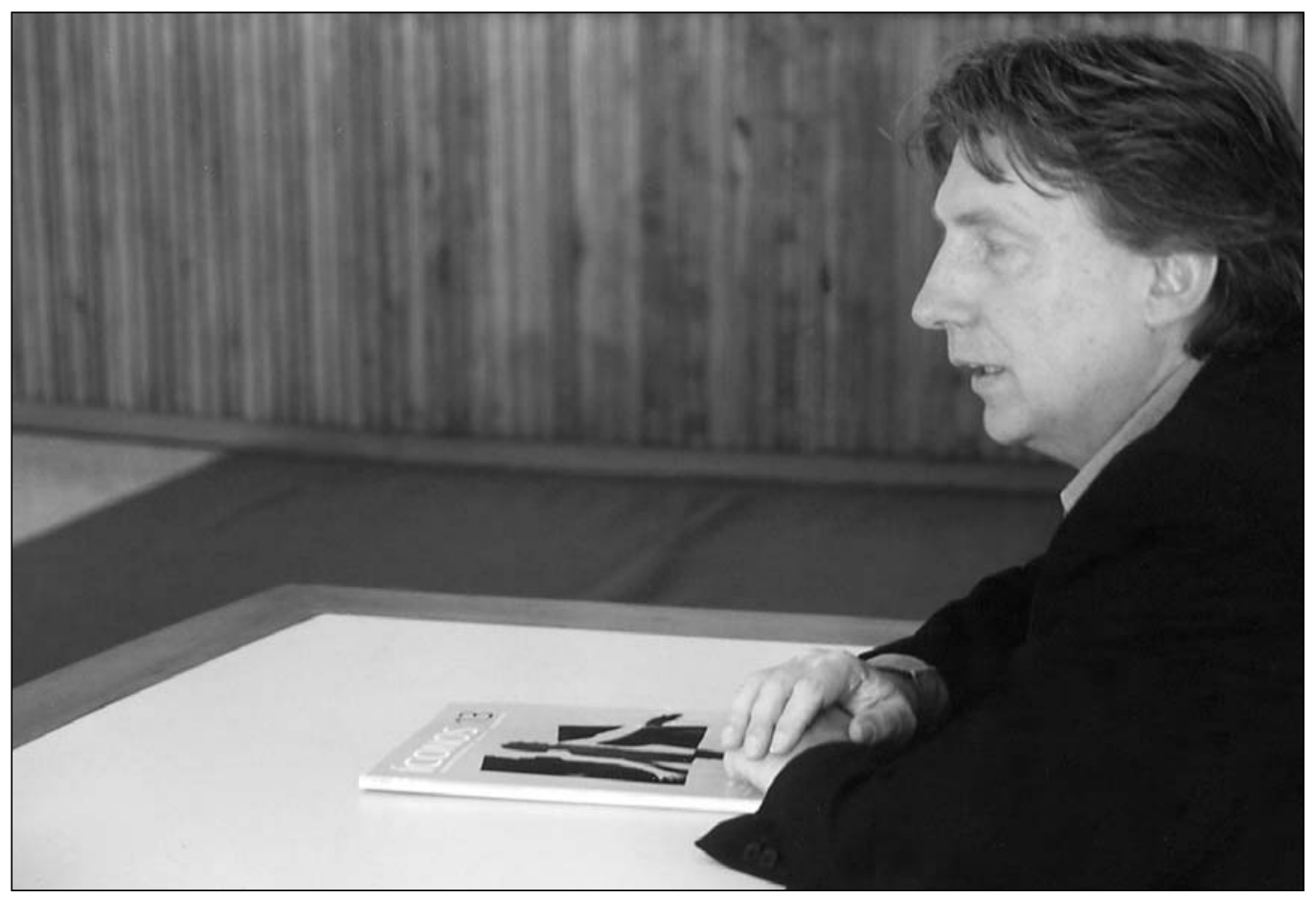

no de obra calificada. Ello en muchos casos, se produjo a costa del empleo de los trabajadores con menos educación, quienes cayeron en el desempleo o tuvieron que buscar trabajo en el sector informal y, generalmente, perdieron remuneración. En otras palabras, la liberalización comercial ha sido buena para algunos y no tanto para otros. El efecto sobre la pobreza es menos generalizable porque ésta también ha sido fuertemente influenciada por otros factores, tales como la política macroeconómica. Si se aísla sólo el impacto de la liberalización comercial, se puede decir que en muchos casos ha sido un elemento que contribuyó con el aumento de la pobreza, si no se lo contrarresta con otros factores, que sí resultan favorables.

F.F. - M.L.G: ¿Existen diferencias entre los distintos países, o se puede afirmar que el impacto ha sido homogéneo?

Aires: Ediciones Universidad de Buenos Aires. También será publicado en inglés: Rob Vos, Lance Taylor y Ricardo Paes de Barros (eds.) Economic Librealization, Distribution and Poverty. Latin America in the 1990s, Cheltenham (Inglaterra): Edward Elgar.
La tendencia hacia una mayor desigualdad entre trabajadores calificados y no calificados se observa en casi toda la región. Sin embargo, el impacto sobre la pobreza y la desigualdad en el ámbito de los hogares no ha sido uniforme; tampoco lo han sido los mecanismos de ajuste estructural. Por ejemplo, en Argentina, la apertura tanto comercial como financiera ha otorgado un empuje al aumento de la productividad en el sector manufacturero, lo cual ha producido una expulsión de trabajadores tanto calificados como no calificados.

Inicialmente ello no provocó un mayor crecimiento de la pobreza, gracias a la expansión y modernización del sector de servicios (telecomunicaciones, etc.), donde el empleo se expandió, sobre todo para la mano de obra calificada. Ya mucho antes de la crisis actual, la suerte de los argentinos se revirtió, proceso que se inició con el efecto "Tequila", ante la crisis que sufriera el peso mexicano en 1995.

Con la disminución de los flujos de capital a Argentina se frenó también la expansión del empleo en el sector de servicios y el desempleo aumentó significativamente. La tendencia estructural ha tendido a que el aumen- 
to de la desigualdad y la pobreza en Argentina de los 90, se deba principalmente al desempleo estructural generado en el sector manufacturero, y al aumento de la brecha de las remuneraciones entre trabajadores calificados y no calificados en el sector de servicios. La crisis actual ha multiplicado dicho efecto sobre la pobreza, particularmente a través del rápido aumento del desempleo y la reducción de los salarios reales.

En México, en cambio, la liberalización comercial y la apreciación del peso mexicano a inicios de los 90, impulsaron una modernización del sector manufacturero, con mayor demanda de mano de obra calificada a costa de los trabajadores de menor calificación. Los trabajadores con menos educación tuvieron cierta suerte con la expansión de la maquila, pero muchos tuvieron que buscar empleo en el sector informal urbano o en la agricultura tradicional. Igualmente, aumentó la desigualdad pero a causa de otro tipo de ajuste estructural en la economía.

En algunos países con mayor peso de la maquila o en otros sectores orientados a la exportación se consiguió generar buena cantidad de nuevos empleos, especialmente para las mujeres. Éste ha sido el caso en países como El Salvador, República Dominicana o Chile. Allí, las mejoras en los niveles de remuneración y la disminución del desempleo ayudaron a reducir la pobreza. Una lección que nos queda a partir de estos casos radica en que se aplicaron esquemas de promoción de exportaciones (no tradicionales) relativamente eficaces y amplios.

En Ecuador se observa también un aumento de la demanda de mano de obra calificada después de la liberalización comercial, a inicios de los años 90; pero igual, en casi todas las ramas de actividad con excepción de los sectores de comercio y servicios personales donde se encuentra la mayoría del sector informal urbano que aparece como el empleador residual de la economía. Tanto antes como después de la crisis de 1999 se observa un aumento de la desigualdad en los ingresos laborales en el país, que se contrarresta sólo momentáneamente en aquellos años en que se aplicaron ajustes significativos en los salarios mínimos.

F.F. - M.L.G: ¿Cuál es la importancia relativa y la secuencia de elementos como estabilización, crecimiento $y$ distribución en una estrategia sostenible de desarrollo económico y social?

Es difícil establecer prioridades entre estos elementos porque están relacionados en gran medida. Sin estabilidad es difícil lograr un crecimiento sostenido, mientras el crecimiento con mucha desigualdad tiende a enfrentar límites como la falta de expansión de un mercado interno, demasiada tensión social o mucha gente sin acceso a la educación. Si se proyecta la pregunta al caso ecuatoriano, la estabilidad económica es una condición necesaria para crear el contexto que permita encaminar acciones que ayuden a promover la inversión y el crecimiento sostenido en el largo plazo. Así, se daría una alta prioridad a asegurar la estabilidad económica. Una de las formas más estructurales para reducir la desigualdad y sostener un proceso de crecimiento alto radica en la inversión en el desarrollo humano, es decir en educación, salud, etc. Ésta debe ser otra prioridad y requeriría, entre otros elementos, que se otorgase mayor preponderan- 
cia al gasto social en el presupuesto del Estado a costa de otros gastos, si se busca resguardar la estabilidad macroeconómica.

Considero que en algunas otras áreas también se requiere conceder cierta prioridad a las políticas redistributivas, sobre todo a la redistribución de activos mediante una reforma agraria en ciertas partes del sector rural y mayor acceso a créditos para la pequeña industria, por ejemplo. El crecimiento más bien debe ser visto como un resultante de estas condiciones antes que como un objetivo de las políticas en sí mismas.

F.F. - M.L.G: ¿Mantiene aún relevancia la denominada "economía del desarrollo" en el contexto económico internacional actual?

Indudablemente que sí. Una de las características importantes del enfoque de la economía del desarrollo desde sus fundamentos teóricos es que toma en consideración los impedimentos estructurales para el desarrollo. Son aspectos que en la actualidad se tienden a denominar como "factores institucionales". Pueden cubrir muchos componentes, desde el ámbito legal del funcionamiento de mercados, la regulación de mercados financieros, la credibilidad de las intervenciones del gobierno, los arreglos en los mercados financieros, etc., etc. Estos aspectos que tienen sus propias características en determinados espacios geográficos son obvios en el contexto de muchos países en vías de desarrollo.

Aunque desde ángulos distintos, la economía del desarrollo ha puesto a veces énfasis en la importancia así como en el contexto histórico de estos factores, la relevancia actual obvia es que en los contextos de globalización y liberalización económica se siente con mucha más fuerza si los arreglos institucionales son débiles o inadecuados para "controlar" el funcionamiento de los mercados, lo cual presenta efectos desastrosos como los que hemos observado en Ecuador, Argentina y en muchos otros lugares del continente. No es gratuito que ya no exista economista en el FMI o el Banco Mundial que desconozca la impor- tancia crucial de estos factores, aunque a veces la retórica suene diferente o la práctica de las políticas malinterprete estos elementos que no se dejan reformar fácilmente.

Es más bien la Economía tradicional neoclásica, predominante en muchas universidades de los países ricos, que debería aprender mucho de la denominada "economía del desarrollo".

También en las economías "desarrolladas" importan mucho las instituciones. Poco a poco se acepta esta posición en la Economía ortodoxa. Por ejemplo, la teoría de la información asimétrica y los arreglos de garantías implícitas a contratos de préstamo, tal como fue desarrollada por Stiglitz, constituye ya el enfoque estándar en la teoría del funcionamiento de mercados financieros con resultados muy distintos al supuesto tradicional de mercados flexibles con información perfecta. El mismo Stiglitz formó muchas de sus ideas teóricas (tal vez no sus ideas sobre el FMI) en Kenia, en un contexto con problemas de desarrollo, y ahora sus propuestas son muy aceptadas entre los economistas neoclásicos.

La teoría neoclásica de crecimiento endógeno que surgió en los años 80 , constituye un ejemplo distinto. Aquí se trata de explicar los factores que a su vez aclaran el cambio tecnológico detrás del crecimiento de largo plazo, incluidos los factores institucionales. Cuando uno estudia bien las primeras teorías del desarrollo observa que en gran medida, la teoría neoclásica de crecimiento endógeno trata de incorporar los mismos elementos enfatizados ya en las ideas planteadas hace más de cincuenta años por los teóricos del desarrollo, aunque ahora se las estudie con más herramientas matemáticas y econométricas. Lastimosamente, muchos economistas neoclásicos no tienden a reconocer estas contribuciones y pretenden haber inventado la rueda.

F.F. - M.L.G: ¿Cuáles son las corrientes ideológicas relevantes en el debate sobre la economía del desarrollo?

En décadas pasadas había una controversia fuerte entre una tendencia llamada "estructu- 


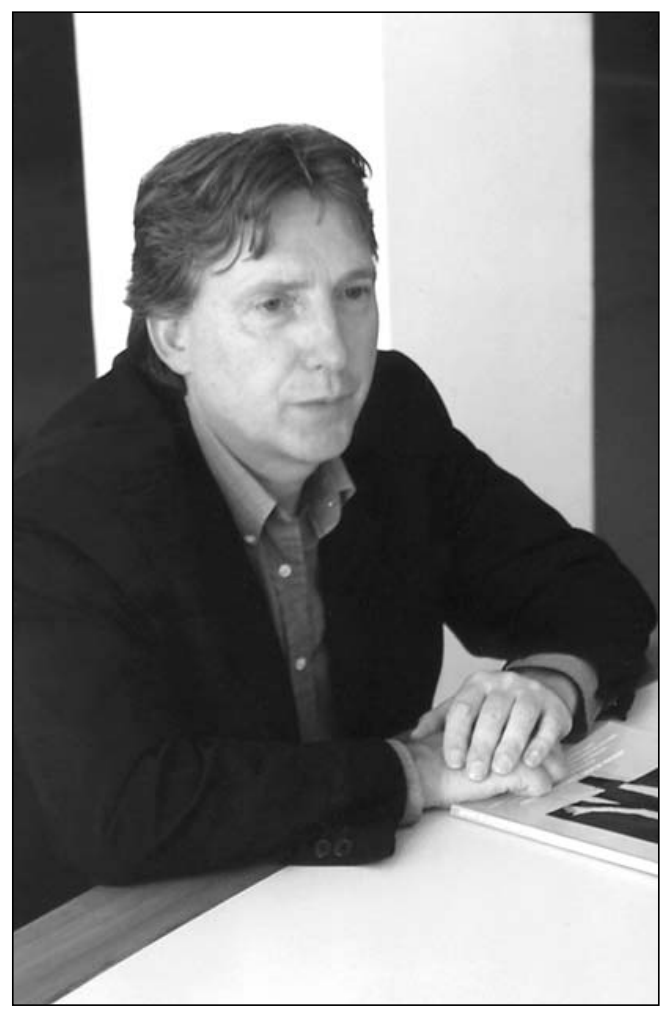

ralista" (que incluye la teoría de la dependencia) y la "neoclásica". Entre los economistas del desarrollo, el estructuralismo (por cierto con diferentes orientaciones) fue más bien la "ortodoxia", expresada en ese entonces, y entre otros aspectos, en la política de sustitución de importaciones, que para bien o para mal, tuvo un fundamento teórico fuerte en el pensamiento de Raúl Prebisch y la CEPAL. Pero también predominó, créanme o no, una escuela estructuralista en el Banco Mundial en los años 60 y 70, cuando Hollis Chenery era economista jefe $y$, entre otros factores, fue protagonista de una estrategia de desarrollo denominada "crecimiento con redistribución”; enfatizaba en que sin reducción de la desigualdad y redistribución de activos no es posible lograr un crecimiento sostenible de la economía.

La "contrarrevolución” neoclásica, si se quiere, surgió sobre todo en los 80 , y declaró la bancarrota de los modelos anteriores, tanto teóricos como de políticas de desarrollo. Las deficiencias de la intervención estatal recomendada por los estructuralistas serían mucho peores que las deficiencias de los mercados. Así surgió la filosofía sustentada por el pensamiento neoclásico en cuanto a que es mejor reducir la intervención estatal y liberalizar la economía. Estamos viviendo este proceso ahora. Sin embargo, como indiqué anteriormente, la teoría neoclásica ya no es tan simple en cuanto a sus supuestos sobre la realidad, como tal vez sugieren los libros de texto: se han incorporado muchos de los elementos enfatizados por los estructuralistas. En el ámbito teórico creo que ahora hay mucha más convergencia entre las dos posiciones.

También los "estructuralistas" reconocen que no se puede suponer tanta rigidez en el funcionamiento de las economías en desarrollo, tal como la ineficacia de los mecanismos de ajuste de los precios relativos, por ejemplo. La diferencia crucial tiende a ubicar cuál es el punto de partida para el análisis. Comenzamos con una situación "ideal" de mercado y el tema central es estudiar "las distorsiones" de dicho mundo ideal, como suele ser la formación de muchos economistas neoclásicos; o más bien partimos de un contexto histórico de mercados imperfectos, agentes que no siempre pueden "optimizar" su utilidad, e instituciones que pueden funcionar de manera distinta en contextos diferentes.

Creo que el punto de partida debe ser la última posición, pero con el reconocimiento de que tanto de la escuela neoclásica como de otras escuelas podemos derivar las herramientas de análisis que nos permitan entender mejor la realidad y guiar la política económica. Las posiciones ideológicas también influyen en el pensamiento económico, pero lo peor es caer en posiciones fundamentalistas. Esto no ayuda ni al avance teórico, ni al desarrollo económico.

F.F. - M.L.G: Usted se ha dedicado a investigar la realidad económica y social del Ecuador desde inicios de la década de los 80 . ¿Cuál es el balance económico y social que haría sobre los últimos veinte años en el país?

Si se observan los años recientes se diría obviamente, que el balance ha sido más bien ne- 
gativo. Sin embargo, quisiera enfatizar primero en ciertos logros que no se reconocen a veces. El desarrollo social ha mejorado indudablemente en algunos aspectos. La esperanza de vida ha aumentado en las últimas dos décadas de 60 a 70 años, la escolaridad de la población en general ha mejorado sustancialmente, la tasa de mortalidad infantil bajó de 50 a 18 por cada mil nacidos vivos, y también hoy existe mucha más gente con acceso al agua potable que hace veinte años. Todos éstos son logros importantes que hay que reconocer. En general, si se lo mide a través de estos indicadores sociales, los ecuatorianos viven mejor hoy que hace dos décadas. No obstante, también existen razones para una visión más pesimista:

- Primero, se observa un cierto estancamiento en los logros sociales durante la última década, a causa, en parte, de la caída casi perpetua del gasto social real desde 1982 .

- Segundo, se ha dado demasiada volatilidad en la situación económica que ha causado mucha incertidumbre y ha frenado inversiones que realmente contribuyen al desarrollo a largo plazo. Dicha volatilidad no solo se debe a la mala suerte, como en el caso del fenómeno El Niño o las caídas en el precio del petróleo. Más bien, el país ha sufrido además, de un mal manejo de la economía, sin mucha visión de largo plazo. Sabemos que el precio del petróleo es inestable y que hay muchas formas para suavizar el impacto de esta volatilidad. No se han aplicado, y hasta la actualidad faltan, las medidas para un manejo prudente de la riqueza generada por el petróleo. No se puede evitar que ocurra el fenómeno El Niño, pero sí hay varias formas para prevenir muchos de los daños que causa típicamente. En este sentido, se ha realizado poco esfuerzo real. El manejo de la política económica y social en las últimas décadas ha estado sujeto a los ciclos políticos y a los intereses particulares. En el panorama político actual no he escuchado voces que realmente apunten seriamente a superar esta deficiencia.
- Tercero, en términos políticos e institucionales el país ha caído en una crisis de gobernabilidad. La gente tiene poca confianza en lo que hace el Gobierno, pero al mismo tiempo, exige que éste resuelva todos los problemas. No hay medidas milagrosas que puedan revertir esta situación de un día para el otro. Va a ser necesario un proceso duro y largo para superar esto.

\section{F.F. - M.L.G: ¿Es partidario de la dolarización?}

Me han hecho muchas veces esta pregunta. Creo que no está adecuadamente formulada. ¿Por qué? Porque es difícil favorecer un régimen monetario frente a otro, sin ver las condiciones bajo las cuales se lo propone. Es un error creer que con un cambio de la moneda se resuelven automáticamente los problemas. En Ecuador, a inicios del año 2000, existió una crisis económica y financiera y sobre todo también una crisis de confianza en el manejo de la economía que requería un shock monetario de algún orden. Con el alto grado de dolarización de facto (con la gente teniendo ya muchos dólares en su "Colchon Bank"), una dolarización oficial era una opción no tan ilógica. En teoría, creo que para un país como Ecuador no conviene un régimen monetario tan inflexible porque disminuye considerablemente las opciones para ajustar la economía ante desequilibrios económicos sin afectar mucho el bienestar de la población. Sin embargo, en la actualidad - ya tomada la decisión de dolarizar - creo que es inconveniente proponer revertirla. ¿Cuánto mejor ha vivido el país con los regímenes cambiarios de los años 80 ó 90 ? Creo que revocar el esquema de dolarización ahora, tendría un costo económico y social tremendo por la incertidumbre que causaría y por la historia del mal manejo monetario que el país ha experimentado en los últimos veinte años o más.

Es más, el debate sobre dolarizar o no, desvía la atención de los problemas de mayor envergadura que es necesario resolver. La dolarización por sí misma no trae automáticamente 
más estabilidad, ni tampoco su eliminación. El país puede caer nuevamente en una crisis financiera tanto bajo la dolarización como sin ella. La ventaja principal de mantener ahora la dolarización radica en que ofrece un punto fijo de confianza al sistema económico, que debería ayudar a pensar sobre otras reformas que son necesarias bajo cualquier régimen monetario, tales como crear un fondo de estabilización y ahorro para los recursos petroleros, introducir un sistema de manejo fiscal sostenible, profundizar la reforma a la regulación y supervisión bancaria, entre otros aspectos.

Sin un manejo mejor en estas áreas, la economía puede hundirse bajo cualquier régimen monetario. Es demasiado fácil apuntar a la dolarización como el mal de la economía. Considero que ahora hay que aprovechar el hecho de que la dolarización ha simplificado las opciones de manejo macroeconómico para realizar las reformas que se debieron haber hecho hace décadas.

F.F.- M.L.G: En el ámbito de las próximas elecciones presidenciales, ¿Cuáles considera Ud. que son los desafíos de política económica y social en el contexto de la dolarización de la economía ecuatoriana?

Como lo mencioné anteriormente, considero que en primer lugar, es necesario dejar de lado la discusión sobre la dolarización. Hay que apuntar al tipo de reformas económicas que he señalado. En el ámbito social se requiere una agenda tan ambiciosa como realista. Primero, hay que revertir la tendencia hacia la reduc- ción del gasto social de los últimos veinte años. Este cambio ya se ha iniciado bajo el gobierno de Gustavo Noboa. No obstante, requiere un esfuerzo sostenido que, con las limitaciones fiscales dadas, implica una clara prioridad por mucho tiempo para el gasto público en educación, salud y protección social, algo que ha faltado en todos los gobiernos anteriores. Segundo, hay que mejorar mucho la calidad y la eficiencia del mismo gasto para lograr mejores resultados. Tercero, se requiere fortalecer en gran medida el sistema de protección social. La crisis de 1999-2000 mostró que ni el sistema de seguridad social ni los programas de asistencia social tenían la cobertura y la flexibilidad para dar protección a la población más vulnerable, justamente cuando más se lo necesitaba. En muchos hogares pobres, algunos niños dejaron de ir a la escuela o sufrieron de desnutrición, lo que afectará sus posibilidades a una vida mejor en el largo plazo.

En una economía dolarizada el gobierno tiene menos opciones de manejo económico a corto plazo para suavizar dichos costos sociales, por lo que requiere de un sistema de protección social que pueda ser más efectivo en períodos de revés económico y que ahorre recursos en tiempos mejores. No es fácil realizar este tipo de cambios en una situación de restricción fiscal, pero hay que caminar en esta dirección y asumir una visión que, ojalá, supere el ciclo político y que sea durable. En el pasado hemos visto demasiados procesos de un paso hacia delante y tres para atrás, tal como en un tango falso. Hay que intentar otro baile que busque sólo los pasos pa’ delante. 